\title{
Fast Local Binary Pattern: Application to Document Image Retrieval
}

\author{
Fahimeh Alaei \\ School of ICT, Griffith \\ University, Australia \\ fahimeh.alaei@griffithuni.edu.au
}

\author{
Alireza Alaei \\ School of ICT, Griffith \\ University, Australia \\ alireza20alaei@yahoo.com
}

\author{
Umapada Pal \\ CVPR Unit, Indian \\ Statistical Institute, India \\ umapada@isical.ac.in
}

\author{
Michael Blumenstein \\ University of Technology \\ Sydney, Australia \\ Michael.Blumenstein@uts.edu.au
}

\begin{abstract}
The volume of digitised documents is increasing every day. Thus, designing a fast document image retrieval method for the large volume of document images, especially when the document images are also large in size, is of high demand. As feature extraction is one of the important steps in every document image retrieval system, a feature extraction technique with a low computing time and small feature number has a direct effect on the speed of the retrieval system. In this paper, we propose a non-parametric texture feature extraction method based on summarising the local grey-level structure of the image. To extract the proposed features, the input image is, at first, divided into a set of overlapping patches of equal size. The peripheral pixels of the centre pixel in a patch are used to extract two sets of patterns. The patterns are derived from the vertical \& horizontal, and diagonal \& off-diagonal pixels of the patch, separately. From each set of pixels, $15\left(=2^{\wedge} 4-1\right)$ different local binary patterns are extracted in our proposed feature extraction method. Two histograms of the local binary patterns obtained from these two sets of pixels are then created and concatenated to obtain 30 features called fast local binary patterns (F-LBP). To evaluate the efficiency of the proposed feature extraction method, MTDB and ITESOFT databases were considered for experimentation. The proposed F-LBP provided promising results with lower computing time as well as smaller memory space consumption.
\end{abstract}

Keywords: document image retrieval; texture features; local binary pattern

\section{INTRODUCTION}

In any given region of an image, small repeated patterns or the spatial arrangement of pixels can be described as a texture feature [1]. Texture features are able to provide important information about the structural arrangement of pixels and their connections to the adjacent area in an image [2]. Different texture feature extraction methods in the literature can be categorised into four groups including: statistical, structural, model-based, and transform-based approaches [3]. Since statistical based texture extraction methods have provided better accuracy at a reasonable computational cost, they have become more popular among other categories of texture feature approaches [4].

In the literature of pattern recognition, and computer vision, texture features have widely been used in different applications [5-7]. Various feature extraction techniques have also been developed for document image analysis and retrieval [8-10]. The advantages and beneficial uses of texture features for document image retrieval have further been discussed in $[11,12]$.

In [11], a local binary pattern (LBP) method, as a statistical approach, has been used for document image retrieval. The effectiveness of texture features has been explored using document image databases. Likewise in [12], the LBP and discrete wavelet transform (DWT) features have been extracted from each document image to characterize document images. A score level fusion strategy has then been proposed to obtain the final retrieval results in the proposed document image retrieval system [12].

In [13], different texture features, such as grey level cooccurrence matrix (GLCM), Gabor filters, autocorrelation function, and a variety of wavelet transforms, have been compared for segmenting graphical parts of historical documents from textual parts. The authors have stated that the Gabor-based features provided the best result for distinguishing textual regions from graphical ones [13]. In addition, Gabor descriptors have been applied to enhance historical document image segmentation [14].

From the literature review it can be noted that most of the texture feature extraction techniques, such as Gabor and wavelet based features, are computationally expensive [13, 14]. Considering the volume and size of document images, a fast feature extraction method would be of great interest for document image retrieval applications. As extracting effective texture feature has a direct impact on the performance of different document image analysis problems, the purpose of this paper is to propose a new local binary pattern which is more efficient compared to the previous local binary pattern methods in relation to time complexity and memory consumption. Two different databases, MTDB and ITESOFT, are further considered to evaluate the efficiency of the proposed feature extraction method for document image retrieval.

The rest of the paper is organized as follows. In Section II, the proposed F-LBP is explained. Applications of the proposed F-LBP to document image retrieval are discussed in Section III. Detailed experimental results with different analysis as well as comparison analysis of the proposed method with various LBP-based methods are demonstrated in Section IV. Finally, conclusions and future work are presented in Section V. 


\section{PROPOSED FAST LOCAL BINARY PATTERN}

The LBP has been proposed as a means of summarising local grey-level structure for texture classification in [18]. Several modified versions of LBP have also been undertaken to overcome the drawbacks of the LBP method and to enhance the performance of different systems [1, 4, 19, 20]. The applicability of various LBP-based feature extraction methods has also been shown in different applications [7, 11, 12, 15-17].

In [1], to improve the feature extraction method, the local intensity of pixels based on three directions in the neighbourhood of the centre pixel in each patch has been used for image retrieval. To acquire more information from each pixel, the magnitude pattern has also been considered. Similarly, in [21] a local tri-directional weber pattern has been used for face image retrieval. The mutual relationships of the current pixel in three directions with its adjacent neighbourhood pixels were considered. Based on the magnitude of differential excitation, the relationship among the neighbourhood pixels was encoded. As a result, a feature vector of length 512 features was extracted from each image. The texture feature has further been used for image classification in [4]. Pair-wise comparison of pixels along with a closed path around the central pixel was provided on different patterns in a $3 \times 3$ window. In another study, to solve the problem of the sensitivity to noise, a local ternary pattern [15] has been proposed.

The attempts of previous researchers were to enhance the performance or to improve the discriminative ability of the LBP method. The method's final performance was considerably affected by the selection of appropriate neighbourhoods. This selection includes number, size and position of the neighbourhood and the distribution of the sampling points [6]. Since the neighbouring pixels can provide more information compared to the centre pixels, the neighbourhood pixels have usually been considered in the previous works. Our proposed method has an advantage over the previous methods. Here, very little memory space is required since the feature length is reduced compared to the other methods when the value of eight peripheral pixels around the centre pixel is taken into account.

In the original LBP feature extraction method [5], differences between the grey values of a centre pixel, say $c$, of each patch and $N$ neighbouring pixels are measured. The difference value is then encoded with a binary value of 0 or 1 . If the difference is a negative value, the encoding value is considered as 0 and otherwise as 1 . These binary values are concatenated in a clockwise direction to obtain a binary pattern of 8 bits. The decimal value of the binary number is generated and used for labelling the centre pixel. Let $S$ represents a matrix of $3 \times 3$ which is indexed by neighbour pixels of the centre pixel. Corresponding to the different binary patterns $L B P_{N, R}$ produces $2^{N}$ output values.

$$
\begin{aligned}
S & =\left[\begin{array}{lll}
i_{7} & i_{6} & i_{5} \\
i_{0} & i_{c} & i_{4} \\
i_{1} & i_{2} & i_{3}
\end{array}\right] \\
L B P_{N, R} & =\sum_{n=0}^{N-1} 2^{n} * S\left(i_{n}-i_{c}\right)
\end{aligned}
$$

$$
s\left(i_{n}-i_{c}\right)= \begin{cases}1 & \text { if }\left(i_{n}-i_{c}\right) \geq 0, \\ 0, & \text { otherwise }\end{cases}
$$

where $N$ is the number of neighbours of the centre pixel $c, R$ is the radius, and the grey-level values at $c$ and $n$ are represented by $i_{c}$ and $i_{n}$. The concept of uniform pattern is further used in the LBP method to reduce the number of features. A binary pattern is called a uniform binary pattern if the number of transactions between 0 and 1 of the pattern sequence is less than or equal to two.

The improved local binary pattern (ILBP) was later proposed in [19] to reduce the effect of noise in image. In the ILBP operator, the local average grey-level value is considered instead of a centre pixel value as a threshold. Thus, the grey-level neighbourhood pixel values are thresholded by the average grey-level value. The ILBP features have been computed as follows:

$$
\begin{gathered}
I L B P=2^{n} * f\left(i_{c}-M\right) \sum_{n=0}^{N-1} 2^{n} * f\left(i_{n}-M\right) \\
f\left(i_{c, n}-M\right)= \begin{cases}1 & \text { if }\left(i_{c, n}-M\right) \geq 0, \\
0, & \text { otherwise }\end{cases} \\
M=\frac{1}{9}\left(i_{c}+\sum_{n=0}^{7} i_{n}\right)
\end{gathered}
$$

where $M$ is considered as the average value of a precise patch. In the ILBP method, $2^{9}-1$ binary patterns have been produced.

\begin{tabular}{|l|l|l|}
\hline$i_{7}$ & $i_{6}$ & $i_{5}$ \\
\hline$i_{0}$ & $i_{c}$ & $i_{4}$ \\
\hline$i_{1}$ & $i_{2}$ & $i_{3}$ \\
\hline
\end{tabular}

(a)

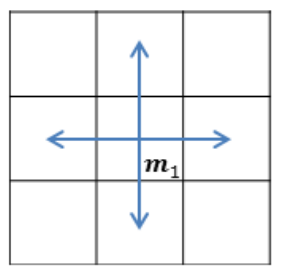

(b)

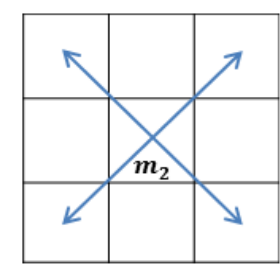

(c)
Fig. 1. (a) Arrangement of a $3 \times 3$ matrix, (b) layout of the fast local pattern using vertical \& horizontal pixels, (c) layout of the fast local pattern using diagonal \& off-diagonal pixels

Inspired by the ILBP, a new version of the LBP called Fast LBP (F-LBP) is proposed in this research work. The proposed F-LBP performs well and faster in document image retrieval compared to the LBP and ILBP methods. Moreover, compared to the LBP and ILBP methods, the proposed F-LBP method needs smaller memory space as a smaller number of features is extracted for each patch. The positioning and layout of the proposed F-LBP extracted from a patch are represented in Fig. 1. In the LBP and ILBP methods all 8 neighbours of the centre pixel are generally used for computing a binary pattern for a patch of size $3 \times 3$. As a result, $256\left(2^{8}\right)$ different patterns from LBP and $511\left(2^{9}-1\right)$ different patterns from ILBP are obtained, and a large feature vector is computed for an image. However, in our proposed FLBP method, the neighbouring pixels are categorised into two groups: vertical \& horizontal pixels, and diagonal \& offdiagonal pixels. Each group includes only 4 neighbouring pixels resulting in $15\left(2^{4}-1\right)$ different binary patterns, which is considerably less than the number of binary patterns generated in the LBP $\left(2^{8}=256\right)$ and $\operatorname{ILBP}\left(2^{9}-1=511\right)$ feature extraction methods. 
The proposed feature extraction method is composed of two parts, including $\mathrm{F}_{1} \mathrm{LBP}$ and $\mathrm{F}_{2} \mathrm{LBP}$. For computing the $\mathrm{F}_{1} \mathrm{LBP}$, the first group of neighbouring pixels is considered. The mean value of the centre pixel and four neighbour pixels located in the horizontal and vertical positions (Fig. 1(b)) of the centre pixel is computed using equation (11). The mean value is considered as a threshold in our proposed F-LBP, whereas in the LBP operator, the value of the central pixel has been considered as the threshold. The differences between the threshold value and the pixels located in the horizontal and vertical positions of the centre pixel are then computed. A binary pattern is obtained employing equation (9) on the obtained difference values, and the corresponding decimal number of the binary value is finally obtained using equation (7). The occurrence frequency of the different binary patterns obtained from the input image is represented using a histogram of 15 bins. Subsequently, in the second part, to compute the $\mathrm{F}_{2} \mathrm{LBP}$ operator, the pixels located on the diagonal and off-diagonal positions of the centre pixel are taken into account. The mean value of the centre pixel and other pixels located in the diagonal and offdiagonal positions is obtained. The diagonal pixels are shown in Fig. 1(c). Similar to the $F_{1}$ LBP operator, using equations (8), (10), and (12), the same procedure is applied to obtain another histogram of 15 bins.

$$
\begin{gathered}
F_{1} L B P=\sum_{n=0}^{n \in \text { even }} 2^{n} * s\left(i_{n}-m_{1}\right), \quad n<8 \\
F_{2} L B P=\sum_{n=0}^{n \in \text { odd }} 2^{n} * s\left(i_{n}-m_{2}\right), \quad n<8
\end{gathered}
$$

In the final step, two histograms $F_{1} L B P$ and $F_{2} L B P$ are concatenated to obtain F-LBP feature of size 30. As a result, the F-LBP method could summarise the local greylevel structure in each patch using less memory space as well as less computing time compared to the other variations of the LBP method. A thorough experimentation is provided in the subsequent section to demonstrate the efficiency of the proposed F-LBP with respect to time complexity and retrieval accuracy.

\section{APPLICATION TO DOCUMENT IMAGE RETRIEVAL}

In this research work, we employed the proposed F-LBP operator in a conventional document image retrieval system. The block diagram of the document image retrieval used in this research work is presented in Fig. 2. It is composed of training and testing phases. Each phase follows preprocessing, and feature extraction steps used commonly in every retrieval system. A brief description of each phase is presented below in the form of a pseudo code algorithm.

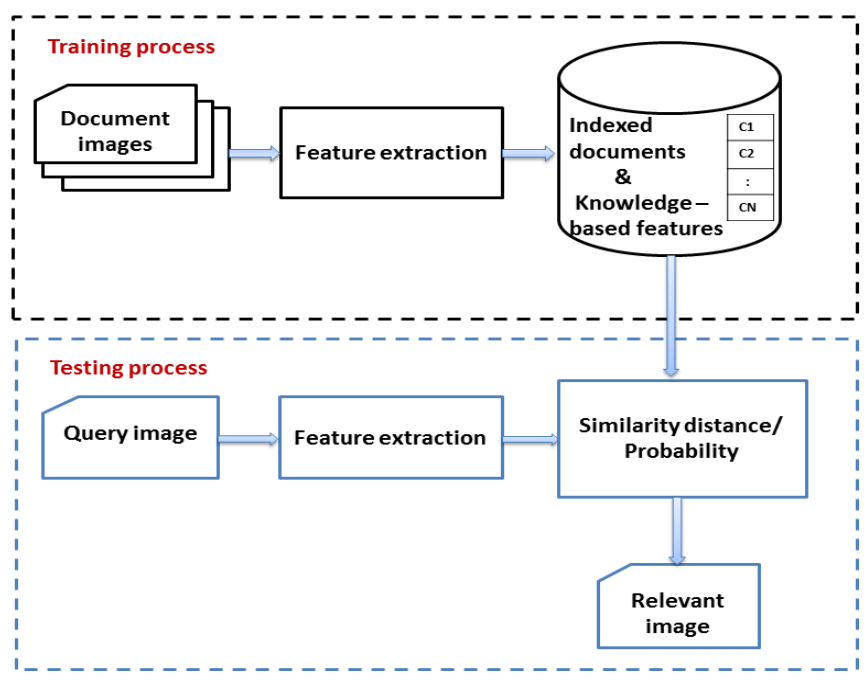

Fig. 2. Block diagram of the document image retrieval process

\section{Phase 1: Training}

Input: Document images used for training

Output: Indexed document and create knowledge-based features.

1. Pre-processing: convert the input images into grey scale.

2. Extract the features from each document image using the proposed F-LBP.

3. Create a knowledge based information.

\section{Phase 2: Testing}

Input: A query image

Output: Rank the images to be shown to the user.

1. Pre-processing: convert the input images into grey scale.

2. Extract the features from a query document image using the proposed F-LBP.

3. Compute the similarity distance between the features extracted from the given query document image and the features stored in the knowledgebase.

4. Rank the document images based on maximum visual similarity to the given query image.

In our proposed document image retrieval system, nearest neighbour-based methods are used to measure the similarity between a query image and the knowledge-based features. The Tanimoto, Euclidean and City-block distances provided in the following are considered for experimentation in this research work, and the results are compared.

$$
\begin{gathered}
d_{T}(A, B)=\frac{\sum_{1}^{p}(A . B)}{\sum_{1}^{p}\left|A^{2}\right|+\sum_{1}^{p}\left|B^{2}\right|+\sum_{1}^{p}(A . B)} \\
d_{E}(A, B)=\sqrt{\sum_{1}^{p}(A-B)^{2}} \\
d_{C}(A, B)=\sum_{1}^{p}|A-B|
\end{gathered}
$$


where $d_{T}(A, B)$ is the Tanimoto distance, $d_{E}(A, B)$ represents the Euclidean distance, and $d_{C}(A, B)$ computes the City-block distance between feature vectors $A$ and $B . A$ and $B$ are the extracted feature vectors from a query image and a document image from the database, respectively. The number of features in each feature vector is $p$ here.

\section{EXPERIMENTAL RESULTS AND ANALYSIS}

\section{A. Database and Evaluation Metric}

To evaluate the proposed feature extraction method, two different document image databases called Media Team Document Database (MTDB) and ITESOFT were used for experimentation. The MTDB is composed of heterogeneous document images [22]. This database includes a great diversity of page layouts with three different resolutions. In our experiment on the MTDB database, only 154 images (one sample image from each group) were used for training the proposed system, and the remaining 1,168 images were considered for testing.

The ITESOFT database [23] contains document images which were manually categorised into 12 different classes. Each class is composed of document images with two different resolutions: 200 DPI and 300 DPI. The document images are not equally distributed in all the classes of the ITESOFT database. A total of 120 document images (only 10 samples from each class) were considered for training the proposed system, and the remaining 996 images were used for testing purposes. To demonstrate the efficiency of the proposed F-LBP in relation to the computing time, large size images with 300DPI resolution were considered for our experiments.

The evaluation of the system was carried out using the percentage of accuracy as the evaluation metric. The accuracies were measured based on the first (Top-1), Top-3, Top-5, and Top-10 documents which had maximum visual similarity to a given query document image. The Top-1 value represents the accuracy of the system when our document image retrieval system could rank the correct document image with respect to a given query at first place. Similarly, the Top10 value represents the percentage of the correct retrieval results when our document image retrieval system could rank the correct document image with respect to a given query within the first 10 places.

\section{B. Results and Discussion}

The percentages of correct document image retrieval obtained from our proposed method on the MTDB and ITESOFT databases are presented in Tables I and II, respectively. The results were computed based on three different distances.

From the results presented in Table I, can be seen that almost $70 \%$ of queries were accurately ranked within the top 10 MTDB documents when the City-block was used for computing the similarity measures. Table II demonstrates that employing the proposed document image retrieval using the F-LBP feature extraction method on the ITESOFT database, approximately $83 \%$ of queries could be accurately ranked within the top 10 documents with the City-block similarity measure.
As we were interested in the computing time of the proposed F-LBP method, document images of large size with high-resolution from the MTDB and ITESOFT databases were selected for experiments. The size of these document images in high-resolution were approximately of $2800 \times 1700$ and $3700 \times 2500$ pixels in the MTDB and ITESOFT databases. The F-LBP required on average 0.7632 and 1.0653 seconds for extracting features from each document image in the MTDB and ITESOFT databases, respectively. To compare and get an idea of the required computing time for extracting features, various LBP-based feature extraction method were considered for experimentation and the results are discussed in the following section.

TABLE I. THE RETRIEVAL RESULTS OBTAINED USING OUR PROPOSED METHOD ON THE MTDB.

\begin{tabular}{lcccc}
\hline Result & $\begin{array}{c}\text { Top-1 } \\
(\boldsymbol{\%})\end{array}$ & $\begin{array}{c}\text { Top-3 } \\
(\boldsymbol{\%})\end{array}$ & $\begin{array}{c}\text { Top-5 } \\
(\boldsymbol{\%})\end{array}$ & $\begin{array}{c}\text { Top-10 } \\
(\boldsymbol{\%})\end{array}$ \\
\hline Tanimance & 58.73 & 66.61 & 67.98 & 69.52 \\
Euclidean & 58.65 & 66.44 & 68.49 & 69.35 \\
City-block & 58.99 & 65.84 & 68.41 & 69.98 \\
\hline
\end{tabular}

TABLE II. THE RETRIEVAL RESULTS OBTAINED USING OUR PROPOSED METHOD ON THE ITESOFT DATABASE.

\begin{tabular}{lcccc}
\hline Result & $\begin{array}{c}\text { Top-1 } \\
\text { Distance }\end{array}$ & $\begin{array}{c}\text { Top-3 } \\
(\boldsymbol{\%})\end{array}$ & $\begin{array}{c}\text { Top-5 } \\
(\boldsymbol{\%})\end{array}$ & $\begin{array}{c}\text { Top-10 } \\
(\boldsymbol{\%})\end{array}$ \\
\hline Tanimoto & 39.36 & 55.22 & 67.97 & 81.33 \\
Euclidean & 39.36 & 55.22 & 68.17 & 81.82 \\
City-block & 42.17 & 59.14 & 67.17 & 82.93 \\
\hline
\end{tabular}

\section{Comparative Analysis}

To show the efficiency of our proposed F-LBP feature extraction method, various LBPs and the ILBP were used for feature extraction in our document retrieval process for comparison. These methods were applied on the MTDB and ITESOFT databases and the results are demonstrated in Tables III and IV. Furthermore, to compare different LBPbased feature extraction methods and our proposed F-LBP, the number of features and the computing times obtained from the F-LBP and various LBP-based methods are shown in Fig. 3 (a-b).

From the results shown in Table III, it can be noted that the proposed F-LBP feature extraction method provides better document image retrieval compared to the other LBP-based methods reviewed in this research work. The same conclusion can also be drawn from the results presented in Table IV in relation to the ITESOFT database. Higher improvements, up to $2 \%$ in document image retrieval accuracy, are obtained by F-LBP compared to other LBP-based methods. It is worth mentioning that this improvement was achieved using a smaller number of features as well as reducing computing time compared to all other LBP-based feature extraction methods except $\mathrm{LBP}_{4,1}$ (Fig. 3). 
TABLE III. THE RETRIEVAL RESULTS OBTAINED USING THE LBP VARIATIONS ON THE MTDB.

\begin{tabular}{|c|c|c|c|c|c|c|c|c|c|c|c|c|c|c|}
\hline \multirow[b]{2}{*}{ Methods } & \multirow[b]{2}{*}{$\begin{array}{l}\text { Computing } \\
\text { Time }\end{array}$} & \multirow[b]{2}{*}{$\begin{array}{c}\text { No. of } \\
\text { Features }\end{array}$} & \multicolumn{4}{|c|}{ Tanimoto Distance } & \multicolumn{4}{|c|}{ Euclidean Distance } & \multicolumn{4}{|c|}{ City-block Distances } \\
\hline & & & $\begin{array}{c}\text { Top-1 } \\
(\%)\end{array}$ & $\begin{array}{c}\text { Top-3 } \\
(\%)\end{array}$ & $\begin{array}{c}\text { Top-5 } \\
(\%)\end{array}$ & $\begin{array}{c}\text { Top-10 } \\
(\%)\end{array}$ & $\begin{array}{c}\text { Top-1 } \\
(\%)\end{array}$ & $\begin{array}{c}\text { Top-3 } \\
(\%)\end{array}$ & $\begin{array}{c}\text { Top-5 } \\
(\%)\end{array}$ & $\begin{array}{c}\text { Top-10 } \\
(\%)\end{array}$ & $\begin{array}{c}\text { Top-1 } \\
(\%)\end{array}$ & $\begin{array}{c}\text { Top-3 } \\
(\%)\end{array}$ & $\begin{array}{c}\text { Top-5 } \\
(\%)\end{array}$ & $\begin{array}{c}\text { Top-10 } \\
(\%)\end{array}$ \\
\hline LBP & 0.9882 & 256 & 53.25 & 64.98 & 67.07 & 69.26 & 57.28 & 65.12 & 67.38 & 69.09 & 58.99 & 67.72 & 68.18 & 69.16 \\
\hline $\mathrm{LBP}_{4,1}$ & 0.6946 & 16 & 57.11 & 64.92 & 67.47 & 69.18 & 57.19 & 64.52 & 67.72 & 69.09 & 57.79 & 66.52 & 67.47 & 68.92 \\
\hline $\mathbf{L B P}_{8, \mathbf{1}}$ & 1.5323 & 59 & 57.28 & 64.73 & 67.98 & 69.43 & 57.18 & 65.49 & 67.89 & 69.35 & 58.48 & 67.21 & 68.58 & 69.15 \\
\hline $\mathbf{L B P}_{12,1.5}$ & 3.3937 & 135 & 57.11 & 64.58 & 67.38 & 69.01 & 58.22 & 64.90 & 67.98 & 69.01 & 58.76 & 67.89 & 68.49 & 69.18 \\
\hline $\mathbf{L B P}_{16,2}$ & 5.0382 & 243 & 56.85 & 64.58 & 66.95 & 68.75 & 56.93 & 64.04 & 67.21 & 68.92 & 57.93 & 67.72 & 68.18 & 69.09 \\
\hline ILBP & 1.0471 & 511 & 50.67 & 52.23 & 55.22 & 59.85 & 56.85 & 65.41 & 67.98 & 69.09 & 57.19 & 65.61 & 68.66 & 69.43 \\
\hline F-LBP & 0.7632 & 30 & 58.73 & 66.61 & 67.98 & 69.52 & 58.65 & 66.44 & 68.49 & 69.35 & 58.99 & 66.84 & 68.41 & 69.98 \\
\hline
\end{tabular}

TABLE IV. THE RETRIEVAL RESULTS OBTAINED USING THE LBP VARIATIONS ON THE ITESOFT DATABASE.

\begin{tabular}{|c|c|c|c|c|c|c|c|c|c|c|c|c|c|c|}
\hline \multirow[b]{2}{*}{ Methods } & \multirow[b]{2}{*}{$\begin{array}{l}\text { Computing } \\
\text { Time }\end{array}$} & \multirow[b]{2}{*}{$\begin{array}{c}\text { No. of } \\
\text { Features }\end{array}$} & \multicolumn{4}{|c|}{ Tanimoto Distance } & \multicolumn{4}{|c|}{ Euclidean Distance } & \multicolumn{4}{|c|}{ City-block Distances } \\
\hline & & & $\begin{array}{c}\text { Top-1 } \\
(\%)\end{array}$ & $\begin{array}{c}\text { Top-3 } \\
(\%)\end{array}$ & $\begin{array}{c}\text { Top-5 } \\
(\%)\end{array}$ & $\begin{array}{c}\text { Top-10 } \\
(\%)\end{array}$ & $\begin{array}{c}\text { Top-1 } \\
(\%)\end{array}$ & $\begin{array}{c}\text { Top-3 } \\
(\%)\end{array}$ & $\begin{array}{c}\text { Top-5 } \\
(\%)\end{array}$ & $\begin{array}{c}\text { Top-10 } \\
(\%)\end{array}$ & $\begin{array}{c}\text { Top-1 } \\
(\%)\end{array}$ & $\begin{array}{c}\text { Top-3 } \\
(\%)\end{array}$ & $\begin{array}{c}\text { Top-5 } \\
(\%)\end{array}$ & $\begin{array}{c}\text { Top-10 } \\
(\%)\end{array}$ \\
\hline LBP & 1.0812 & 256 & 34.54 & 52.91 & 63.86 & 80.42 & 34.44 & 52.91 & 63.96 & 80.42 & 40.46 & 60.74 & 71.39 & 80.62 \\
\hline $\mathbf{L B P}_{4,1}$ & 1.0449 & 16 & 34.24 & 54.02 & 67.07 & 80.12 & 34.24 & 54.12 & 67.17 & 80.12 & 37.25 & 59.74 & 70.58 & 81.12 \\
\hline $\mathbf{L B P}_{8,1}$ & 2.3718 & 59 & 33.63 & 53.01 & 65.76 & 81.02 & 33.53 & 53.01 & 65.86 & 81.33 & 38.05 & 58.84 & 70.08 & 81.63 \\
\hline $\mathbf{L B P}_{12,1.5}$ & 5.6693 & 135 & 34.84 & 50.30 & 65.36 & 80.02 & 34.84 & 50.60 & 65.36 & 80.02 & 40.56 & 58.03 & 68.27 & 82.53 \\
\hline $\mathbf{L B P}_{16,2}$ & 7.3688 & 243 & 35.24 & 50.20 & 65.56 & 79.82 & 39.66 & 58.94 & 67.98 & 81.85 & 41.06 & 61.75 & 74.70 & 82.85 \\
\hline ILBP & 1.1159 & 511 & 37.55 & 53.11 & 63.96 & 80.12 & 37.65 & 53.21 & 63.86 & 80.12 & 41.67 & 60.24 & 67.77 & 82.83 \\
\hline F-LBP & 1.0653 & 30 & 39.36 & 55.22 & 67.97 & 81.33 & 39.36 & 55.22 & 68.17 & 81.82 & 42.17 & 59.14 & 67.17 & 82.93 \\
\hline
\end{tabular}

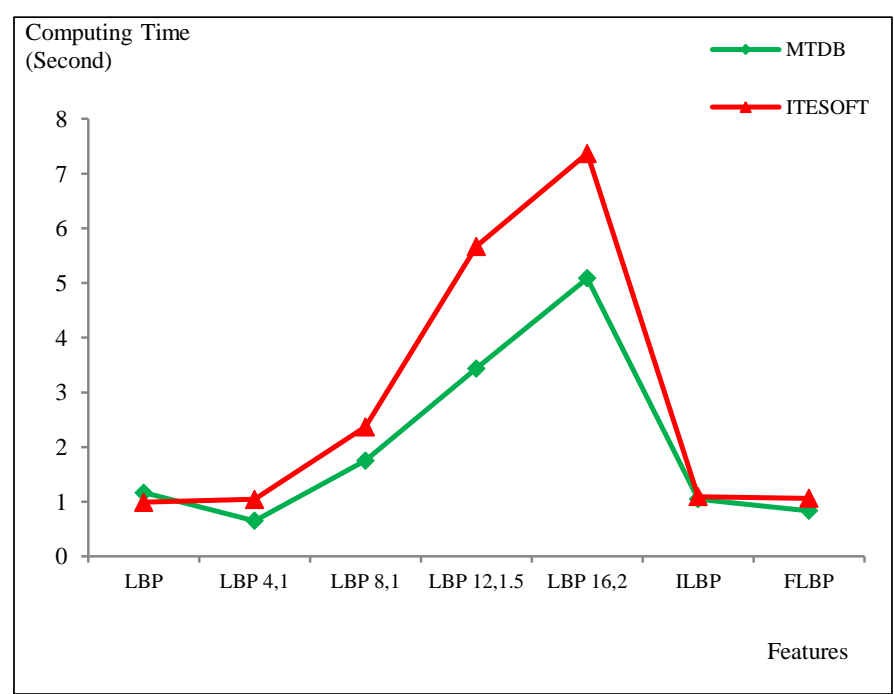

(a)

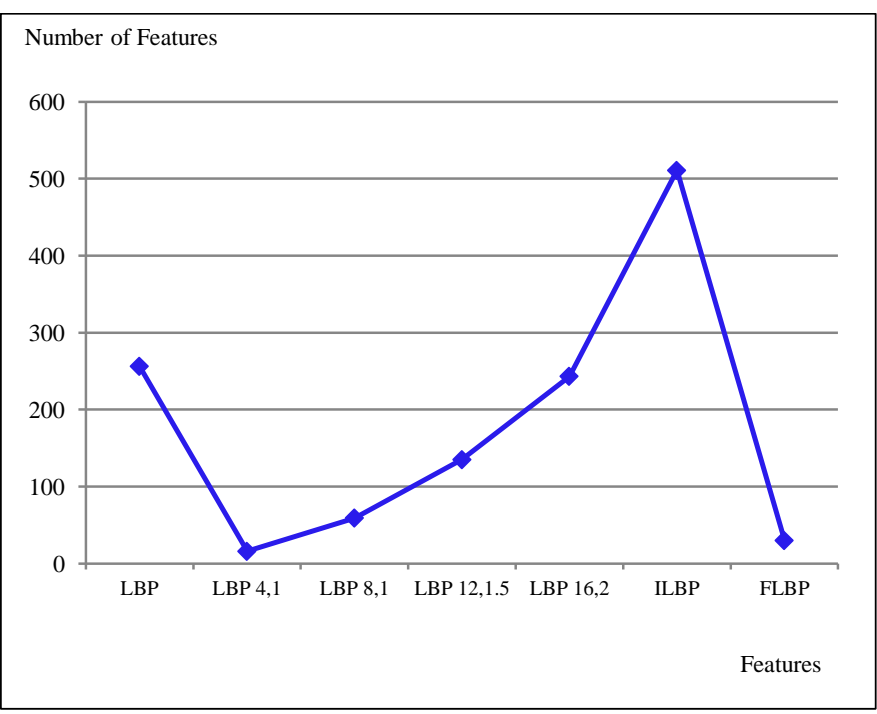

(b)

Fig. 3. (a) Graph showing computing time for different LBPs and the proposed F-LBP, (b) graph showing number of extracted features for different LBPs and the proposed F-LBP 
The computing time in the proposed F-LBP can be further reduced by using parallel programming, as the $F_{1} L B P$ and $\mathrm{F}_{2} \mathrm{LBP}$ can be developed using parallel implementation. This finding has important implications for developing a more efficient system for collection of images of a large size.

\section{V.CONCLUSIONS AND FUTURE WORK}

In this paper, we have proposed a fast local binary pattern method for feature extraction towards document image retrieval. The spatial arrangement of the grey-level of the pixels in local patches of an image is considered for feature extraction. Since the neighbouring pixels have been grouped into two categories, two separate texture features, one from each group, have been computed for each local patch. The features have then been concatenated to obtain the final FLBP feature vector of size 30. The accuracy of correct document image retrieval was satisfactory. The proposed FLBP feature extraction method required less computing time and less memory space to extract the features from the document image compared with most of the LBP-based feature extraction techniques discussed in the literature.

In future, we plan to extend the proposed F-LBP using bigger size patches as we have used only a $3 \times 3$ patch size in this research work. We also plan to use parallel programming techniques for implementing the proposed algorithm to reduce the computing time even further. We hope such fast LBP will be helpful to other researchers for applications to image retrieval.

\section{ACKNOWLEDGMENT}

The authors would like to thank V.P. D'Andecy of ITESOFT Company for providing access to the ITESOFT database.

\section{REFERENCES}

[1] M. Verma, and B. Raman, "Local tri-directional patterns: A new texture feature descriptor for image retrieva," Digital Signal Processing, 51: pp. 62-72, 2016.

[2] R.M. Haralick, K. Shanmugam, and I.H. Dinstein, "Textural features for image classification," IEEE Tarns. On Systems, Man, and Cybernetics, 3(6): pp. 610-621, 1973

[3] H.B. Manish, J.J. Liu, and J. F. MacGregor, "Image texture analysis: methods and comparisons," Chemometrics and Intelligent Laboratory Systems, 72(1):pp. 57-71. 2004.

[4] A. Fernández, M.X. Álvarez, and F. Bianconi, "Image classification with binary gradient contours," Optics and Lasers in Engineering, 49(9): pp. 1177-1184, 2011

[5] T. Ojala, M. Pietikäinen, and T. Mäenpää "Multiresolution gray-scale and rotation invariant texture classification with local binary patterns," IEEE Trans. Pattern Analysis and Machine Intelligence, 24(7): pp. 971-987, 2002.

[6] D. Huang, C. Shan, M. Ardabilian, Y. Wang, and L.Chen, "Local binary patterns and its application to facial image analysis: a survey", IEEE Trans. Systems, Man, and Cybernetics, 41(6): pp. 765-781, 2011.

[7] J.F. Vargas, M.A. Ferrer , C.M. Travieso, and J.B. Alonso "Off-line signature verification based on grey level information using texture features," Pattern Recognition, 44(2): pp. 375-385, 2011.

[8] S. Marinai, "Page Similarity and Classification," Handbook of Document Image Processing and Recognition, pp. 223-253, 2014

[9] J. Kumar, P. Ye, and D. Doermann, "Structural similarity for document image classification and retrieval," Pattern Recognition Letters, 43: pp. 119-126, 2014

[10] K. Kise, W. Yin, and K. Matsumoto. "Document image retrieval based on 2D density distributions of terms with pseudo relevance feedback," In Proceedings of the International Conference on Document Analysis and Recognition, pp. 488-492, 2003.

[11] F. Alaei, A. Alaei, U. Pal, and M. Blumenstein "Document Image Retrieval Based on Texture Features: A Recognition-Free Approach,"
In Proceedings of the International Conference on Digital Image Computing: Techniques and Applications, pp.1-7, 2016.

[12] F. Alaei, A. Alaei, M. Blumenstein, and U. Pal, "Document image retrieval based on texture features and similarity fusion," In Proceedings of the International Conference on Image and Vision Computing New Zealand, pp. 1-6, 2016.

[13] M. Mehri, M. Mhiri, P. Héroux, J.K. Petra, M.A. Mahjoub, and R. Mullot,"Performance evaluation and benchmarking of six texturebased feature sets for segmenting historical documents," In Proceedings of the International Conference on Pattern Recognition, pp. 2885-2890, 2014.

[14] M. Mehri, N. Nayef, P. Héroux, J.K. Petra, and R. Mullot, "Learning Texture Features for Enhancement and Segmentation of Historical Document Images," In Proceedings of the International Workshop on Historical Document Imaging and Processing, pp. 47-54, 2015.

[15] X. Tan, and B. Triggs, "Enhanced local texture feature sets for face recognition under difficult lighting conditions," IEEE Trans. Image Processing, 19(6): pp. 1635-1650, 2010.

[16] M. Pietikäinen,T Nurmela, T. Mäenpää, and M. Turtinen, "Viewbased recognition of real-world textures," Pattern Recognition, 37(2): pp. 313-323, 2004

[17] M. Heikkilä, and M. Pietikäinen, "A texture-based method for modeling the background and detecting moving objects," IEEE Trans. Pattern Analysis and Machine Intelligence, 28(4): pp. 657-662, 2006.

[18] T. Ojala, M. Pietikäinen, and D. Harwood, "A comparative study of texture measures with classification based on featured distributions," Pattern Recognition, 29(1): pp. 51-59, 1996.

[19] H. Jin, Q. Liu, H. Lu, and X. Tong, "Face detection using improved LBP under bayesian framework," In Proceedings of the International Conference on Image and Graphics, pp. 306-309, 2004

[20] L. Nanni, A. Lumini, and S. Brahnam, "Local binary patterns variant as texture descriptors for medical image analysis," Artificial Intelligence in Medicine, 49(2): pp. 117-125, 2010.

[21] V.S.K. Gangavarapu, and G.K.M. Pillutla, "Local tri-directional weber patterns: A new descriptor for texture and face image retrieval," In Proceedings of the International Journal of Computer Science and Information Technologies, 7 (3), pp. 1571-1577, 2016.

[22] J. Sauvola, and H. Kauniskangas, "MediaTeam document database II. A CD-ROM collection of document images," University of Oulu, Finland, 1999

[23] F. Alaei, N. Girard, S. Barrat, J.-Y. Ramel, "A New One-Class Classification Method Based on Symbolic Representation: Application to Document Classification", In Proceedings of the Document Analysis Systems, pp. 272-276, 2014. 